\title{
A Novel Hypoxic Inc-RNA, HRL-SC, Promotes Proliferation and Migration of Human Dental Pulp Stem Cells Through PI3K/AKT Signalling Pathway
}

\section{Junkai Zeng}

Southern Medical University Nanfang Hospital

\section{Ming Chen}

Stomatological Hospital of Southern Medical University

\section{Yeqing Yang}

Stomatology Hospital of Southern Medical University

Buling Wu ( $\sim$ bulingwu@smu.edu.cn )

Shenzhen Hospital of Southern Medical University https://orcid.org/0000-0002-7931-1009

\section{Research Article}

Keywords: Human dental pulp stem cells, Hypoxia, IncRNA, Proliferation and migration

Posted Date: December 13th, 2021

DOI: https://doi.org/10.21203/rs.3.rs-1143046/v1

License: (a) (i) This work is licensed under a Creative Commons Attribution 4.0 International License. Read Full License 


\section{Abstract}

Background: Human dental pulp stem cells (hDPSCs) are critical for pulp generation. hDPSCs proliferate faster under hypoxia, but the regulatory mechanism of long noncoding RNAs (IncRNAs) in this process is not fully understood.

Methods: Novel IncRNAs were obtained by reanalysis of transcriptome datasets coming from RNA-Seq under hypoxia compared with normoxia, and differential expression analysis of target genes were performed. Bioinformatics analyses including Gene Ontology (GO) analysis, Kyoto Encyclopedia of Genes and Genomes (KEGG) pathway analysis and Gene Set Enrichment Analysis (GSEA) analysis were used to understand the function of key novel IncRNA. hDPSCs were isolated from dental pulp tissue. EdU test and scratch healing test were used to detect the proliferation and migration of hDPSCs. qRT-PCR was used to detect the RNA level expression changes of selected genes. RNA fluorescence in situ hybridization (FISH), small interfering RNA (siRNA), qRT-PCR and western blot analysis were used to explore the function of key novel IncRNA.

Results: We identified 496 novel IncRNAs in hDPSCs under hypoxia, including 45 expressed differentially novel IncRNAs. Of them, we focused on a key novel IncRNA, which we named HRL-SC (hypoxia related IncRNA in stem cells). Functional annotation revealed that HRL-SC was associated with hypoxic conditions and PI3K/AKT signalling pathway. HRL-SC was mainly located in the cytoplasm of hDPSCs and had stably high expression under hypoxia. Knockdown of HRL-SC inhibited proliferation and migration of hDPSCs and expression levels of PI3K/AKT related marker proteins. Furthermore, AKT activator SC79 partially offset the inhibitory effect caused by the knockdown, indicating that HRL-SC promoted hDPSCs through PI3K/AKT signalling pathway.

Conclusion: Hypoxia related IncRNA HRL-SC promotes proliferation and migration of hDPSCs through $\mathrm{PI} 3 \mathrm{~K} / \mathrm{AKT}$ signalling pathway and it may provide a better understanding for regenerative application of hDPSCs.

\section{Introduction}

hDPSCs are isolated from dental pulp tissues with the capacity of self-renewal and multiple differentiation[1]. hDPSCs are ideal seed cells for dental pulp tissue engineering and have been widely using in pulp regeneration research[2,3].

Oxygen concentration of human organs in physiological conditions are much lower than normal oxygen conditions, vary from 1\%-12\%[4,5]. Dental pulp tissues are surrounded by rigid dentin and apical foramen is the only vascular access. The pulp tissues are also in lower oxygen tension levels[6]. In our previous study[7], we demonstrated that hypoxia enhances the proliferation and migration, consistent with other researches[8-10]. In addition, the increased expression levels of pluripotency markers in hDPSCs, such as SRY-Box transcription factor 2 (SOX2), octamer-binding protein 4 (Oct-4) and c-Myc, were observed under hypoxia[11]. Hypoxic signalling response is orchestrated by Hypoxia Inducible Factor (HIF), activating 
glycolytic enzymes to provide sources of energy for stem cells[12,13]. Furthermore, because hypoxia maintain undifferentiated state and promote proliferation of stem cells, it is required to explore the potential mechanisms for stem cell under hypoxia.

The expression levels of IncRNA, a specific group of non-coding composed of more than 200 nuclieotides, also change under hypoxia[14,15]. IncRNA could bind the functional proteins and regulate gene expression on transcriptional or post-transcriptional level to exert their functions[16]. Increasing evidence indicated that IncRNAs participated in the hypoxia related cellular process of stem cells, including proliferation, migration, differentiation and stemness. Some studies demonstrated the IncRNAs modulated by hypoxia promoted proliferative and migratory capacity of mesenchymal stem cells[17-19]. Some studies demonstrate that IncRNAs are involved in hypoxia-related metabolism and signaling pathway[14,20]. For example, IncRNA-miRNA-mRNA network analysis with IncRNAs as the core components were performed to understand the molecules expression patterns of hypoxia-promoting effects in olfactory mucosa mesenchymal stem cells[17]. It was also revealed that hypoxia-responsive IncRNAs STL may be the key component of a coding-noncoding gene co-expression network which regulated functions of stem cells[20].

However, very few studies have explored the roles of IncRNAs in regulation of hDPSCs, and the mechanisms involved remain largely unclear. Here, we re-analyze our previous RNA-seq data of hDPSCs under hypoxia and normoxia, and differential expression analysis of target gene were performed to under function of novel IncRNAs. We identified a key novel IncRNA HRL-SC that was significantly upregulated in hDPSCs under hypoxia compared with normoxia. Functional annotations revealed that HRL-SC were closely associated with hypoxia signatures. Knockdown of HRL-SC inhibited the proliferation and migration of hDPSCs under hypoxia and decreased the activity of PI3K/AKT signally pathway. But the activators of AKT offset the inhibition caused by knockdown of HRL-SC. Our data show that hypoxiarelated HRL-SC promote cell proliferation and migration of hDPSCs via PI3K/AKT signaling pathway and provide potential molecular mechanism of hDPSCs under hypoxia.

\section{Materials And Methods}

\section{RNA-Seq data processing}

RNA-Seq had been performed for hDPSCs under normoxia and hypoxia in our previous study, which can be obtained from NCBI GEO: GSE118046. Fastqc (version 0.11.9) was used to access the sequencing quality and Trimmomatic was used to filter low-quality sequencing reads. After the quality control, reads were aligned to reference genome (Hg19) using HISAT2 (version 2.1.0) and the sam files for each sample were obtained. Next, sam files were converted to sorted bam files using Samtools (version 1.13). Assembly of bam files and gene quantification (FPKM values) for each sample was using StringTie (version 2.1.5) and the '-merge' function was performed to merge the transcript. 


\section{Identification of novel IncRNA}

The pipeline for identifying novel IncRNA from assembled transcript are as follow: (1) filter transcripts with class code ' $i, j, u, x^{\prime}$ from Gffcompare of StringTie; (2) remove the transcripts that matched annotations of known IncRNA; (3) filter transcripts with length $\geq 200 \mathrm{bp}$, exon numbers $\geq 1$; (4) filter transcripts without coding potential using the protein-coding gene prediction tools including Coding-NonCoding-Index (CNCl, version 2), Coding Potential Calculator (CPC, version 2), Coding Potential Assessment Tool (CPAT) and Pfam-scan (version 14.3)[21]. Finally, retaining transcripts of which FPKM > 0 in at least one sample were novel IncRNAs.

\section{Differential expression analysis}

Differential expression analysis was used to identify the key novel IncRNAs under hypoxia. Expression matrixs of FPKM values for mRNA and novel IncRNA were imported into R (version 3.63) and analyzed using Linear Models for Microarray data (limma, https://bioconductor.org/ packages/limma/) package in Bioconductor. Up- or downregulated differentially expressed mRNA/novel IncRNA between samples under hypoxia and normoxia were identified with the cut-off criteria of $\mathrm{P}<0.05$ and $\mid$ fold change $(\mathrm{FC}) \mid>2$.

\section{LncRNA target prediction and functional annotation analysis}

In order to understand the function of key IncRNA, LncTar (version) was used to explore the potential interaction between key IncRNA and differentially expressed genes. LncTar is a bioinformatics tool that can calculate the free energy between IncRNA and target genes and a threshold of - 0.1 ndG (default parameter) was set. Next, the target genes were imported into Database for Annotation Visualization and Integrated Discovery (DAVID) (https://david.ncifcrf.gov/) to perform GO enrichment and KEGG pathway analysis. To further explore the function of IncRNA HRL-SC, GSEA (version 4.1.0) was performed using c2.all.v7.4.symbols.gmt (http://software.broadinstitute.org/gsea/downloads.jsp) as a reference gene set. $\mathrm{P}<0.05$ and FDR $<0.25$ were considered statistically significant.

\section{Cell culture and identification}

The hDPSCs were obtained from the third molar of patients aged 18-25 years indicated for extraction at the Department of Stomatology in Nanfang Hospital, Southern Medical University,Guangzhou, Guangdong, China. All experimental protocols were approved by the Ethical Committee of Southern Medical University. The isolated hDPSCs were cultured in Dulbecco modified eagle's medium (DMEM) supplemented with $10 \%$ fetal bovine serum (FBS; ExCell Bio, Shanghai, China), $100 \mathrm{U} / \mathrm{ml}$ penicillin, and $100 \mu \mathrm{g} / \mathrm{ml}$ streptomycin (Sigma, St. Louis, Mo, USA) at $37^{\circ} \mathrm{C}$ in humidified atmosphere containing $5 \%$ $\mathrm{CO} 2$ with different oxygen concentrations. Induced conditioned medium was used for cell induction and differentiation. The culture media were renewed every 3 days. 
After 21 days of differentiation, Alizarin Red and ALP activity staining were performed to verify the formation of mineral nodes and purple colored precipitate. Besides, the cells were incubated for surface markers including CD45, CD34, CD90, CD44 and CD29 and then were detected by flow cytometry to identify the cell phenotypes of hDPSCs.

\section{Cell transfection}

The siRNAs against IncRNA and control siRNA were obtained from GeneChem (Shanghai, China). The details of sequences could be seen in Additional file 1 Table S1. The siRNAs and control siRNA were transfected using riboFECT ${ }^{\mathrm{TM}} \mathrm{CP}$ (Ribobio, GuangZhou, China) according to the manufacturer's protocol. When SC79

\section{Wound healing assay}

Migration capability of hDPSCs was characterized by wound healing assay. Cells were cultured with DMEM supplement with 10\% FBS in 6-well plates until they reached confluence. After starving for $24 \mathrm{~h}$ with serum-free DMEM, scratches were generated with a 1-mL pipette tip in each well. After scratching for $0 \mathrm{~h}$ (right after scratch), $12 \mathrm{~h}$ and $24 \mathrm{~h}$, cell migration of cells was observed and photographed under inverted microscope. Scratch healing area across each scratch was analyzed calculated by using ImageJ software.

\section{Cell proliferation assay}

Proliferation capability of hDPSCs was measured using the EdU (meilunbio, Dalian, China) assay. The hDPSCs were labeling with EdU for $2 \mathrm{~h}$ and then fixed, washed, permeabilized and stained according to the manufacturer's protocol. After cells washed, the DNA was stained with Hoechst 33342, then each well was observed and photographed under an inverted fluorescent microscope.

\section{RNA extraction and RT-PCR}

Total RNA was extracted from each group using EZ-press RNA Purification Kit (EZBiosicence, USA) according to the manufacturer's protocol and quantified by NanoDrop ND-1000 (Thermo Scientific, USA).

Reverse transcription was performed using Color Reverse Transcription Kit (EZBiosicence, USA) with gDNA remover. A volume of $13 \mu \mathrm{L}$ RNA solution of each sample (containing $1 \mu \mathrm{g}$ RNA) was treated with $2 \mu \mathrm{L}$ provided gDNA remover to remover genomic DNA. Each reaction was set for $20 \mu \mathrm{L}$, including $13 \mu \mathrm{L}$ processed RNA solution and $5 \mu \mathrm{L} 4 \times$ RT Master Mix. 


\section{qRT-PCR validation}

Real time PCR was performed with Color SYBR Green qPCR Master Mix ROX2 plus (EZBiosicence, USA) on Roche LightCycler ${ }^{\circledR} 480$. Each reaction was set for $10 \mu \mathrm{L}: 5 \mu \mathrm{L} 2 \times$ Color Green qPCR Master Mix, $0.2 \mu \mathrm{L}$ Forward Primer, $0.2 \mu \mathrm{L}$ Reverse Primer, 3.6 $\mu \mathrm{L}$ RNase Free $\mathrm{dH} 2 \mathrm{O}$ and $1 \mu \mathrm{L}$ Product from RT reaction. Ten differentially expressed novel IncRNAs, primer sequences of which can be seen in Table. All experiments were repeated three times, and relative gene expression was calculated using the 2- $\Delta \Delta \mathrm{Ct}$ method. The sequences of gene-specific primers are listed in Additional file 1 Table S2.

\section{Western blot analysis}

Cells of each group were lysed using the RIPA lysis buffer with protease inhibitors and the total proteins contents were quantitated by the BCA assay (Beyotime, Shanghai, China). The samples were separated by $8 \%$ SDS-PAGE, and then were transferred onto polyvinylidene diffluoride (PVDF) membranes. The PVDF membranes were blocked in protein free rapid blocking buffer (Epizyme Biomedical Technology, Shanghai, China) for $15 \mathrm{~min}$ and then incubated overnight at $4{ }^{\circ} \mathrm{C}$ with primary antibodies against $\beta$-actin, AKT, p-AKT, PI3K, p-PI3K (Bioss, BeiJing, China), which diluted at 1: 1000. Then, the PVDF membranes were incubated with corresponding secondary antibodies (Proteintech, China). The bands were detected using ECL reagents (Biosharp, China). The densities of protein bands were quantified by image $\mathrm{J}$ software.

\section{Results}

\section{hDPSCs exhibit higher proliferation capacity under hypoxia}

Primary hDPSCs were successfully isolated from dental pulp tissue and passaged (Fig. 1a, b). The results of staining showed that hDPSCs processed multiple differential capacity (Fig. 1c-f). Flow cytometry results showed that hDPSCs were positive for stem cell markers CD44, CD29 and CD90 but negative for hematopoietic cell markers including CD45 and CD34 (Fig. 1g). Then, the migration and proliferation were respectively detected by wound healing assay and EdU assay. As indicated by Fig. 1h-k, hDPSCs proliferated and migrated faster under hypoxia compared with normoxia.

\section{Identification and characterization of novel IncRNA in hDPSCs under hypoxia}

To understand the IncRNAs involved in the process, the RNA-Seq data was re-analyzed. According to pipeline (Fig. 2a), novel IncRNAs that may play an important role in regulating stemness of hDPSCs under hypoxia were obtained. Compared to the references in the GENCODE database, 16498 protein coding genes, 1351 known IncRNA. According to the exon number and transcript length, we further filtered and acquired 1600 potential novel IncRNA transcripts. Then, the coding potential of transcripts was analyzed by CPC, CNCl, CPAT and Pfam-scan, and finally 496 novel IncRNAs that met the definition of IncRNA were obtained (Fig. 2b). Briefly, the novel IncRNAs shows longer transcript length, fewer exons, lower coding 
potential and lower FPKM values, consistent with characterization of IncRNA as previously reported (Fig. 2c-e)[22].

\section{Differential Expression Analysis And Validation Of Novel Lncrnas}

A total of 1684 differentially expressed genes (DEGs), 135 differentially expressed known IncRNAs, 45 differentially expressed novel IncRNAs (DENLs) were screened under hypoxia compared with normoxia, shown in a volcano plot and heatmap (Fig. 3a-c). Details of the Differential expression analyses are provided in Additional file 3. The differential expression of novel IncRNAs was validated by RT-qPCR. The expression levels of novel IncRNAs calculated from RT-qPCR results were similar to those from sequencing data (Fig. 3d). Among them, was stably upregulated under hypoxia compare with normoxia. Therefore, we treated MSTRG.20649.1 as the key novel IncRNA that may regulate stemness of hDPSCs under hypoxia, named it as IncRNA HRL-SC (hypoxia related IncRNA in stem cells).

\section{Identification, Location And Expression Pattern Analysis Of Lncrna Hrl-sc}

IncRNA HRL-SC was located in chromosome 3, from locus 48,529,170 to locus $48,531,178$, consisted of 2 exons and 1 intron (Additional file 2 Fig. 1a). RNA-FISH test was performed to examine the subcellular location of HRL-SC, which showed that HRL-SC was mainly located into the cytoplasm of hDPSCs under hypoxia but in nucleus under normoxia (Fig. 4a). To further understand the expression pattern of HRL-SC in hDPSCs, we detected its expression levels under different oxygen concentrations with different exposure durations. The RT-qPCR results showed that HRL-SC had higher expression level under $3 \%$ oxygen when compared with $1 \%$ oxygen, $5 \%$ oxygen and normoxia (Fig. $4 \mathrm{~b}$ ). In addition, the dynamic expression levels of HRL-SC were also observed in hDPSCs under hypoxia (Fig. 4c).

\section{Target genes and pathway analysis of IncRNA HRL-SC}

To understand the function of key novel IncRNA, the target prediction of HRL-SC was performed, and the results showed that there was a total of 592 potential target genes identified from DEGs. The functions of target genes were analyzed through functional enrichment analysis. As shown in Fig. 5a, target genes of HRL-SC were significantly enriched in response to hypoxia and oxidation-reduction process. GEEA results confirmed this association that the differential expression of HRL-SC was associated with hypoxia conditions (Fig. 5b). In addition, pathway analysis indicated that PI3K/AKT signaling pathway, metabolic pathways and the other related pathway were identified (Fig. 5c). The expression analysis of target genes in pathways were shown in Fig. 5d. Among these, PDK1 was most significantly differentially expressed target genes of in HRL-SC most significant differential expression. (Fig. 5d) The details for prediction of target genes and functional annotation analysis are provide in Additional file 4, 5, 6 . 


\section{Knockdown of IncRNA HRL-SC inhibited the proliferation and migration of hDPSCs}

To confirm the role of HRL-SC in regulating stemness of hDPSCs, siRNA-mediated knockdown was performed and the expression levels of HRL-SC significantly decreased (Additional file 2 Fig. 1b). Moreover, Edu assay showed that EdU labeling cells in siRNA group was significantly less than blank control and siRNA-ctrl group (Fig. 6a). Knockdown of HRL-SC inhibited proliferation of hDPSCs under hypoxia. Furthermore, the knockdown of HRL-SC also inhibited cell migration of hDPSCs. As shown in fig, less migration rate was observed in siRNA group compared with blank control and siRNA-ctrl group (Fig. 6b).

\section{Knockdown of IncRNA HRL-SC inhibited the proliferation and migration of hDPSCs through PI3K/AKT signaling pathway.}

Our previous study demonstrated that PI3K/AKT signaling pathway related marker proteins were increased in hDPSCs under hypoxia. Besides, the expression levels of PDK1, one of the potential target genes of HRL-SC, and of HIF-1a increased during hypoxia exposure but decreased after knockdown of HRL-SC (Fig. 7a-C). Combining with the results from pathway analysis of HRL-SC target genes, we speculated that HRL-SC may regulate stemness of hDPSCs through PI3K/AKT signaling pathway. To test this hypothesis, the expression levels of PI3K/AKT related marker proteins was examined after knockdown of HRL-SC. The results revealed that knockdown of HRL-SC decreased ratios of p-AKT/AKT and p-PI3K/PI3K, preventing the activation of signaling pathway (Fig. 7d-f). Next, we added AKT activator SC79 into the medium when cell transfection performed. The western bolt analysis showed that SC79 enhances AKT phosphorylation and upregulate the expression of p-AKT (Fig. 8a, b). Furthermore, the EdU assay and wound healing assay revealed that SC79 promote proliferation and migration of hDPSCs under hypoxia after knockdown of HRL-SC (Fig. 8c-f). In general, the results demonstrated that HRL-SC regulated the proliferation and migration of hDPSCs under hypoxia through PI3K/AKT signaling pathway.

\section{Discussion}

hDPSCs are usually isolated and cultured under normoxia, different from the stem cell microenvironment[23]. As hDPSCs proliferate during extended culture under normoxia, SenescenceAssociated $\beta-G$ alactosidase levels increased, a premature senescence phenotype of stem cells[24,25]. Injury accumulation caused by high oxygen tension can be damaging to stem cells[26]. On the contrary, low oxygen tension maintains undifferentiated state of hDPSCs and then enhances cell proliferation $[8,9,20]$. Similar results were also observed in our study. When cultured under hypoxia, wound heal assay and edu assay revealed that hDPSCs exhibited higher levels of proliferation and migration compared with normoxia.

In fact, studies about hypoxia-related IncRNAs mostly focused on tumor stem cells[27-29]. For example, a novel IncRNA KB-1980E6.3, was identified from breast cancer stem cells under hypoxia and was proven to maintain the stemness through IncRNA KB-1980E6.3/IGF2BP1/C-Myc axis[29]. The underlying mechanism of hypoxia-related IncRNAs in hDPSCs which also promoted by hypoxia remains unclear. 
In present study, we identified 496 unannotated novel IncRNA from RNA-seq data. Through analyzing the exon number, chromosome distribution, FPKM values and coding ability, we found novel IncRNAs we identified conformed to the basic characteristics of IncRNA. According to the results of differential expression analysis and qRT-PCR, we screened the key novel IncRNA that may play an important function in promoting cell proliferation and migration of hDPSCs under hypoxia. Functions enrichment analysis revealed that HRL-SC was involved in response to hypoxia and the other hypoxia related biological processes. Low oxygen tension was described as physiological oxygen tension inhibits senescence and maintains stem cell properties. Physiological oxygen tension in hypoxic condition is a necessary parameter for activation of hypoxia-inducible factor to promote stemness of stem cells[30,31]. The RNA Fish revealed that HRL-SC located in cell nucleus under normoxia but accumulated in cell cytoplasm under hypoxia. Cytoplasmic IncRNAs directly modulate the stability and translation of mRNA, work as ceRNAs to influence miRNAs and regulate cellular signalling pathway[32]. The increased expression level and changed localization of HRL-SC were observed from normoxia to hypoxia. Combining with the bioinformatic analyses, we demonstrated that HRL-SC worked as a hypoxia related IncRNA to regulate hDPSCs.

To eliminate the functions of HRL-SC in hDPSCs under hypoxia, we used siRNA, an efficient silencing method for cytoplasmic IncRNA[33], to knockdown its expression. Interestingly, knockdown of HRL-SC inhibited both proliferation and migration of hDPSCs under hypoxia, but the negative control siRNA did not cause harmful effects. Furthermore, we observed that one of the enriched pathways was PI3K/AKT signaling pathway which is distinctly pivotal for cell survival and proliferation. Some IncRNAs were demonstrated to regulate activity of stem cells via PI3K/AKT signaling pathway. For example, it was observed that IncRNA AK015322 promote proliferation of hair follicle stem cell via PI3K/AKT signaling pathway[34]. So, we focused on the expression levels of PI3K/AKT related proteins, and found the increased expression of p-AKT and p-PI3K, indicating the activation of PI3K/AKT signaling pathway[35]. Besides, we also observed the increased expression of HIF-1 $a$ and PDK1, one of the potential target gene of HRL-SC. It was demonstrated that HIF-1 a mediate the cellular response to hypoxia[36,37]. LincRNA-p21 was reported as a hypoxia-related IncRNA and enhanced cell migration and survival capacity of MSCs via HIF-1a/CXCR4 and CXCR7 pathway[18]. In hypoxia condition, HIF-1a activates and induces glycolysis providing energy for self-renewal of stem cells[38]. HIF-1a is a key regulator of PDK1 and the inhibition of HIF-1a would significantly reduce the expression of PDK1[39]. Thus PDK1 was considered as a HIF-1adependent gene[40]. More importantly, it is well known that activation of PDK1 can directly phosphorylate AKT at Thr308 activating PI3K/AKT signaling pathway[41]. After knockdown of HRL-SC, the expression levels of p-AKT and p-PI3K decreased, as well as HIF-1 $a$ and PDK1. Similar to our results, knockdown of Inc-ORA, a novel IncRNA that was identified from RNA-Seq data, reduced the expression of p-AKT and p$\mathrm{PI} 3 \mathrm{~K}$ in adipose tissue and in turn Inc-ORA was considered to regulate adipocyte differentiation via PI3K/AKT signaling pathway[22]. Hence, we speculated that HRL-SC promoted hDPSCs through $\mathrm{PI3K} / \mathrm{AKT}$ signaling pathway.

For further validation, we added SC79 into the medium after knockdown. SC79 is one of AKT activators that induces the phosphorylation of AKT to activate PI3K/AKT signaling pathway[42]. And the activation 
of p-AKT caused by SC79 was confirmed. As the results shown, SC79 partially rescued the inhibitory effect of proliferation and migration caused by the knockdown of HRL-SC in hDPSCs under hypoxia. Therefore, we considered that novel HRL-SC promote cell proliferation and migration of hDPSCs under hypoxia via PI3K/AKT signaling pathway.

\section{Conclusion}

In conclusion, we identified a novel HRL-SC from RNA-Seq data of hDPSCs under hypoxia compared with normoxia. HRL-SC is a hypoxia-related IncRNA that promote cell proliferation and migration of hDPSCs through PI3K/AKT signaling pathway. These findings are conducive to understanding of the mechanism for IncRNA in hDPSCs under hypoxia and to the application of hDPSCs in pulp regeneration.

\section{Abbreviations}

$\mathrm{CNCl}$

Coding-Non-Coding-Index

CPAT

Coding Potential Assessment Tool

$\mathrm{CPC}$

Coding Potential Calculator

DAVID

Database for Annotation Visualization and Integrated Discovery

DEGs

differentially expressed genes

DENLS

differentially expressed novel IncRNAs

DMEM

Dulbecco modified eagle's medium

FBS

fetal bovine serum

$\mathrm{FISH}$

fluorescence in situ hybridization

GO

Gene Ontology

GSEA

Gene Set Enrichment Analysis

hDPSCs

Human dental pulp stem cells

HIF

Hypoxia Inducible Factor 
KEGG

Kyoto Encyclopedia of Genes and Genomes

IncRNAs

long noncoding RNAs

Oct-4

octamer-binding protein 4

PVDF

polyvinylidene diffluoride

SiRNA

small interfering RNA

sox2

SRY-Box transcription factor 2

\section{Declarations}

\section{Acknowledgements}

Not applicable.

\section{Authors' contributions}

WBL and ZJK conceived and designed the experiments. ZJK and YYQ contributed to the writing and drafting of the manuscript. ZJK performed the experiments and collected data. ZJK, CM and YYQ analyzed the data. All authors read and approved the final manuscript.

\section{Funding}

This study was supported by the National Natural Science Foundation of China (81870755).

\section{Availability of data and materials}

Not applicable.

\section{Ethics approval and consent to participate}

This study was approved by the Ethics Committee of Nanfang Hospital,

Southern Medical University. Written informed consent was obtained from all

adult patients. For minor population, statement on informed consent was

obtained from their parents.

Consent for publication 
Not applicable.

\section{Competing interests}

The authors declare that they have no competing interests.

\section{References}

1. Gronthos S, Brahim J, Li W, Fisher LW, Cherman N, Boyde A, et al. Stem Cell Properties of Human Dental Pulp Stem Cells. J Dent Res. 2002;81:531-5.

2. Xie Z, Shen Z, Zhan P, Yang J, Huang Q, Huang S, et al. Functional Dental Pulp Regeneration: Basic Research and Clinical Translation. Int J Mol Sci. 2021;22:8991.

3. Yamada Y, Nakamura-Yamada S, Kusano K, Baba S. Clinical Potential and Current Progress of Dental Pulp Stem Cells for Various Systemic Diseases in Regenerative Medicine: A Concise Review. Int J Mol Sci. 2019;20:1132.

4. Csete M. Oxygen in the Cultivation of Stem Cells. Ann N Y Acad Sci. 2005;1049:1-8.

5. Mohyeldin A, Garzón-Muvdi T, Quiñones-Hinojosa A. Oxygen in Stem Cell Biology: A Critical Component of the Stem Cell Niche. Cell Stem Cell. 2010;7:150-61.

6. Yu CY, Boyd NM, Cringle SJ, Alder VA, Yu DY. Oxygen distribution and consumption in rat lower incisor pulp. Arch Oral Biol. 2002;47:529-36.

7. Liu F, Huang X, Luo Z, He J, Haider F, Song C, et al. Hypoxia-Activated PI3K/Akt Inhibits Oxidative Stress via the Regulation of Reactive Oxygen Species in Human Dental Pulp Cells. Oxid Med Cell Longev. 2019;2019:1-10.

8. lida K, Takeda-Kawaguchi T, Tezuka Y, Kunisada T, Shibata T, Tezuka K. Hypoxia enhances colony formation and proliferation but inhibits differentiation of human dental pulp cells. Arch Oral Biol. 2010;55:648-54.

9. Aranha AMF, Zhang Z, Neiva KG, Costa CAS, Hebling J, Nör JE. Hypoxia Enhances the Angiogenic Potential of Human Dental Pulp Cells. J Endod. 2010;36:1633-7.

10. Sakdee JB, White RR, Pagonis TC, Hauschka PV. Hypoxia-amplified Proliferation of Human Dental Pulp Cells. J Endod. 2009;35:818-23.

11. Zhou Y, Fan W, Xiao Y. The Effect of Hypoxia on the Stemness and Differentiation Capacity of PDLC and DPC. BioMed Res Int. 2014;2014:1-7.

12. Vukovic M, Sepulveda C, Subramani C, Guitart AV, Mohr J, Allen L, et al. Adult hematopoietic stem cells lacking Hif-1a self-renew normally. Blood. 2016;127:2841-6.

13. Mathieu J, Zhou W, Xing Y, Sperber H, Ferreccio A, Agoston Z, et al. Hypoxia-Inducible Factors Have Distinct and Stage-Specific Roles during Reprogramming of Human Cells to Pluripotency. Cell Stem Cell. 2014;14:592-605.

14. Xu S, Tang L, Liu Z, Luo C, Cheng Q. Hypoxia-Related IncRNA Correlates With Prognosis and Immune Microenvironment in Lower-Grade Glioma. Front Immunol. 2021;12:731048. 
15. Shi H, Wang M, Sun J, Wang H, Li Y, Chen B, et al. A novel long noncoding RNA FAF inhibits apoptosis via upregulating FGF9 through PI3K/AKT signaling pathway in ischemia-hypoxia cardiomyocytes. J Cell Physiol. 2019;234:21973-87.

16. Mercer TR, Dinger ME, Mattick JS. Long non-coding RNAs: insights into functions. Nat Rev Genet. 2009;10:155-9.

17. He J, Huang Y, Liu J, Ge L, Tang X, Lu M, et al. Hypoxic conditioned promotes the proliferation of human olfactory mucosa mesenchymal stem cells and relevant IncRNA and mRNA analysis. Life Sci. 2021;265:118861.

18. Meng S-S, Xu X-P, Chang W, Lu Z-H, Huang L-L, Xu J-Y, et al. LincRNA-p21 promotes mesenchymal stem cell migration capacity and survival through hypoxic preconditioning. Stem Cell Res Ther. 2018;9:280.

19. Hou J, Wang L, Wu Q, Zheng G, Long H, Wu H, et al. Long noncoding RNA H19 upregulates vascular endothelial growth factor A to enhance mesenchymal stem cells survival and angiogenic capacity by inhibiting miR-199a-5p. Stem Cell Res Ther. 2018;9:109.

20. Shi R, Yang H, Lin X, Cao Y, Zhang C, Fan Z, et al. Analysis of the characteristics and expression profiles of coding and noncoding RNAs of human dental pulp stem cells in hypoxic conditions. Stem Cell Res Ther. 2019;10:89.

21. Mistry J, Chuguransky S, Williams L, Qureshi M, Salazar GA, Sonnhammer ELL, et al. Pfam: The protein families database in 2021. Nucleic Acids Res. 2021;49:D412-9.

22. Cai R, Tang G, Zhang Q, Yong W, Zhang W, Xiao J, et al. A Novel Inc-RNA, Named Inc-ORA, Is Identified by RNA-Seq Analysis, and Its Knockdown Inhibits Adipogenesis by Regulating the PI3K/AKT/mTOR Signaling Pathway. Cells. 2019;8:477.

23. Labedz-Maslowska A, Bryniarska N, Kubiak A, Kaczmarzyk T, Sekula-Stryjewska M, Noga S, et al. Multilineage Differentiation Potential of Human Dental Pulp Stem Cells-Impact of 3D and Hypoxic Environment on Osteogenesis In Vitro. Int J Mol Sci. 2020;21:6172.

24. Alaidaroos NYA, Alraies A, Waddington RJ, Sloan AJ, Moseley R. Differential SOD2 and GSTZ1 profiles contribute to contrasting dental pulp stem cell susceptibilities to oxidative damage and premature senescence. Stem Cell Res Ther. 2021;12:142.

25. Asghari M, Nasoohi N, Hodjat M. High glucose promotes the aging of human dental pulp cells through Wnt/beta-catenin signaling. Dent Med Probl. 2021;58:39-46.

26. Mas-Bargues C, Sanz-Ros J, Román-Domínguez A, Gimeno-Mallench L, Inglés M, Viña J, et al. Extracellular Vesicles from Healthy Cells Improves Cell Function and Stemness in Premature Senescent Stem Cells by miR-302b and HIF-1a Activation. Biomolecules. 2020;10:957.

27. Li J, Liao T, Liu H, Yuan H, Ouyang T, Wang J, et al. Hypoxic glioma stem cell-derived exosomes containing Linc01060 promote progression of glioma by regulating the MZF1/c-Myc/HIF-1a. Cancer Res. 2020;canres.2270.2020.

28. García-Venzor A, Mandujano-Tinoco EA, Ruiz-Silvestre A, Sánchez JM, Lizarraga F, Zampedri C, et al. IncMat2B regulated by severe hypoxia induces cisplatin resistance by increasing DNA damage repair 
and tumor-initiating population in breast cancer cells. Carcinogenesis. 2020;41:1485-97.

29. Zhu P, He F, Hou Y, Tu G, Li Q, Jin T, et al. A novel hypoxic long noncoding RNA KB-1980E6.3 maintains breast cancer stem cell stemness via interacting with IGF2BP1 to facilitate c-Myc mRNA stability. Oncogene. 2021;40:1609-27.

30. Han Y, Gong T, Zhang C, Dissanayaka WL. HIF-1a Stabilization Enhances Angio-/Vasculogenic Properties of SHED. J Dent Res. 2020;99:804-12.

31. Bhandi S, Al Kahtani A, Mashyakhy M, Alsofi L, Maganur PC, Vishwanathaiah S, et al. Modulation of the Dental Pulp Stem Cell Secretory Profile by Hypoxia Induction Using Cobalt Chloride. J Pers Med. 2021;11:247.

32. Tay Y, Rinn J, Pandolfı PP. The multilayered complexity of ceRNA crosstalk and competition. Nature. 2014;505:344-52.

33. Soares RJ, Maglieri G, Gutschner T, Diederichs S, Lund AH, Nielsen BS, et al. Evaluation of fluorescence in situ hybridization techniques to study long non-coding RNA expression in cultured cells. Nucleic Acids Res. 2018;46:e4-e4.

34. Cai B, Zheng Y, Ma S, Xing Q, Wang X, Yang B, et al. Long non-coding RNA regulates hair follicle stem cell proliferation and differentiation through PI3K/AKT signal pathway. Mol Med Rep [Internet]. 2018 [cited 2021 Nov 20]; Available from: http://www.spandidos-

publications.com/10.3892/mmr.2018.8546

35. Xie Y, Shi X, Sheng K, Han G, Li W, Zhao Q, et al. PI3K/Akt signaling transduction pathway, erythropoiesis and glycolysis in hypoxia (Review). Mol Med Rep [Internet]. 2018 [cited 2021 Nov 21]; Available from: http://www.spandidos-publications.com/10.3892/mmr.2018.9713

36. Majmundar AJ, Wong WJ, Simon MC. Hypoxia-Inducible Factors and the Response to Hypoxic Stress. Mol Cell. 2010;40:294-309.

37. Zehra M, Zubairi W, Hasan A, Butt H, Ramzan A, Azam M, et al. Oxygen Generating Polymeric Nano Fibers That Stimulate Angiogenesis and Show Efficient Wound Healing in a Diabetic Wound Model. Int J Nanomedicine. 2020; Volume 15:3511-22.

38. Xiong G, Stewart RL, Chen J, Gao T, Scott TL, Samayoa LM, et al. Collagen prolyl 4-hydroxylase 1 is essential for HIF-1a stabilization and TNBC chemoresistance. Nat Commun. 2018;9:4456.

39. Xu W-N, Zheng H-L, Yang R-Z, Jiang L-S, Jiang S-D. HIF-1a Regulates Glucocorticoid-Induced Osteoporosis Through PDK1/AKT/mTOR Signaling Pathway. Front Endocrinol. 2020;10:922.

40. Xu W-N, Zheng H-L, Yang R-Z, Jiang L-S, Jiang S-D. HIF-1 a Regulates Glucocorticoid-Induced Osteoporosis Through PDK1/AKT/mTOR Signaling Pathway. Front Endocrinol. 2020;10:922.

41. Wang XQ, Lo CM, Chen L, Ngan ES-W, Xu A, Poon RY. CDK1-PDK1-PI3K/Akt signaling pathway regulates embryonic and induced pluripotency. Cell Death Differ. 2017;24:38-48.

42. Luan Q, Pan L, He D, Gong X, Zhou H. SC79, the AKT Activator Protects Cerebral Ischemia in a Rat Model of Ischemia/Reperfusion Injury. Med Sci Monit. 2018;24:5391-7. 


\section{Figures}

\section{Figure 1}

Identification of hDPSCs and promotion of hypoxia on hDPSCs. a, b Primary cultured and passage hDPSCs. Alizarin Red and ALP activity staining was performed and mineral nodes (c) and purple colored precipitate (d) were detected in the induced group but not appeared in non-induced group (e, f). g Surface markers of hDPSCs were detected by Flow cytometric analysis of the cells were positive for CD44, CD29, CD90 and negative for CD45, CD34. h EdU staining analysis of cell proliferation. EdU-positive cells were in red and cell nuclei were dyed in blue. i EdU-positive cell counts in normoxia group vs. hypoxia group. $\mathrm{j}$ Migration capability was characterized by wound healing assay. $\mathrm{k}$ Scratch healing area in normoxia group vs. hypoxia group. ${ }^{\star *} \mathrm{P}<0.01,{ }^{\star \star \star} \mathrm{P}<0.001$

\section{Figure 2}

Identification and characterization of novel IncRNA in hDPSCs under hypoxia. a Pipeline for novel IncRNAs identification. $b$ Venn diagram showed the intersection of coding protein analysis by $\mathrm{CNCl}, \mathrm{CPC}$, CPAT and Pfam-scan. c Transcript length distribution of known IncRNA, novel IncRNA and protein coding gene. $d$ Distribution of exon number in known IncRNA, novel IncRNA and protein coding gene. e Expression level of known IncRNA, novel IncRNA and protein coding gene.

\section{Figure 3}

Differential expression analysis between normoxia and hypoxia group and expression level validation. a Volcano plot of differentially expressed genes, including 1123 upregulated genes and 561 downregulated genes under hypoxia. b Volcano plot of differentially expressed known IncRNAs, including 60 upregulated IncRNAs and 1123 downregulated IncRNAs under hypoxia. c The heatmap of differentially expressed novel IncRNAs. d Expression level of novel IncRNAs in RNA-seq data was validated by qRT-PCR.

\section{Figure 4}

Location and expression pattern analysis of IncRNA HRL-SC. a Subcellular localization of HRL-SC examined by RNA-FISH test under normoxia and hypoxia. FISH probes against HRL-SC were dyed in red by Cy3 and the cell nucleus were dyed in blue by DAPI. b Expression levels of HRL-SC under hypoxia with different exposure durations using qRT-PCR. c Expression levels of HRL-SC under different oxygen concentrations using qRT-PCR. 


\section{Figure 5}

Functional annotation of IncRNA HRL-SC. a Results of biological process from GO analysis. Target genes of HRL-SC enriched in response to hypoxia and oxidation-reduction process. $b$ Enrichment analysis performed with GSEA showed that HRL-SC enriched in hypoxia related pathways. c Signalling pathways in KEGG enrichment analysis. $d$ Expression analysis of target genes in pathways.

\section{Figure 6}

Knockdown of IncRNA HRL-SC inhibited the proliferation and migration of hDPSCs. a EdU staining analysis of cell proliferation before and after knockdown of HRL-SC. EdU-positive cells were in red and cell nuclei were dyed in blue. b EdU-positive cell counts in control group, siRNA-ctrl group and siRNA group. c Wound healing assay was used to analysis migration capability before and after knockdown of HRL-SC. $d$ Scratch healing area in control group, siRNA-ctrl group and siRNA group. ${ }^{\star \star} P<0.01,{ }^{* \star *} \mathrm{P}<$ 0.001

\section{Figure 7}

Knockdown of IncRNA HRL-SC downregulated the related proteins of PI3K/AKT signaling pathway. a, b Western blot analysis showed the expression level of HIF-1 a decreased in the siRNA group. c The expression levels of PDK1 decreased knockdown of HRL-SC using qRT-PCR. d Western blot analysis shows the expression level of p-AKT and p-PI3K decreased in the siRNA group. e, $f$ Densities analysis of protein bands showed decreased ratios of $\mathrm{p}-\mathrm{AKT} / \mathrm{AKT}$ and $\mathrm{p}-\mathrm{PI} \mathrm{KK} / \mathrm{PI} \mathrm{KK} .{ }^{* \star \mathrm{P}}<0.01$, ${ }^{\star \star \star} \mathrm{P}<0.001$

\section{Figure 8}

SC79 partially rescued the inhibitory effect of proliferation and migration caused by the knockdown of HRL-SC. a, b Western blot analysis showed that SC79 increased expression of p-AKT. c EdU staining analysis of cell proliferation before and after addition of SC79. EdU-positive cells were in red and cell nuclei were dyed in blue. $d$ EdU-positive cell counts in control group, siRNA+SC79 group and siRNA group. e Wound healing assay was performed to analysis migration capability before and after addition of SC79. $f$ Scratch healing area in control group, siRNA+SC79 group and siRNA group. ${ }^{*} P<0.05, * \star P<0.01$, $* * * P<0.001$

\section{Supplementary Files}

This is a list of supplementary files associated with this preprint. Click to download.

- Additionalfile1.docx 
- Additionalfile2.docx

- Additionalfile3.xIsx

- Additionalfile4.xlsx

- Additionalfile5.xlsx

- Additionalfile6.xlsx 